\title{
RESUMEN
}

El nuevo desafío de la Educación Superior en Chile está en desarrollar competencias, donde las universidades están llamadas a lograr este fin. Este es el caso de la Universidad de La Serena (ULS) y su Carrera de Auditoría, donde el presente estudio busca evaluar por parte de los empleadores, el desarrollo de competencias profesionales y transversales en 27 estudiantes cohorte 2014 que realizaron su Práctica Profesional el 2017.

Este estudio responde a un enfoque cuantitativo, un diseño no experimental y una investigación descriptivatransversal, donde producto de una encuesta aplicada, se observa una evaluación positiva en el desarrollo de 11 de las 13 competencias consideradas. Donde las competencias transversales, promediaron 3,35 puntos y las profesionales 3,71 puntos de un total de 4,0 puntos, permitiendo inferir, que aunque la mayoría de las competencias fueron bien evaluadas, se debe prestar atención a la adaptación al cambio y a la autonomía e iniciativa personal.

Palabras claves: Competencia profesional, competencia transversal, estudiantes practicantes, empleadores, práctica profesional.

\section{EVALUACIÓN POR LOS EMPLEADORES DEL DESARROLLO DE COMPETENCIAS PROFESIONALES Y TRANSVERSALES EN LOS ESTUDIANTES DE LA CARRERA DE AUDITORIA COHORTE 2014, UNIVERSIDAD DE LA SERENA.}

Cristian Blanco Alfaro ${ }^{1}$ / Patricia Cortes Iturrieta² / Alberto Hernández Venegas ${ }^{3}$

\section{EVALUATION BY THE EMPLOYERS OF THE DEVELOPMENT OF PROFESSIONAL AND TRANSVERSAL COMPETENCES IN THE STUDENTS OF THE AUDIT CAREER COHORT 2014, UNIVERSIDAD DE LA SERENA.}

\section{ABSTRACT}

The new challenge of Higher Education in Chile is to develop competencies, where universities are called to achieve this goal. This is the case of the University of La Serena (ULS) and its Audit Career, where the present study seeks to evaluate by the employers, the development of professional and transversal competences in 27 students from 2014 cohort, who made their Professional Practice in 2017.

This study responds to a quantitative approach, a non-experimental design and a transversal descriptive research, where as a result of an applied survey, a positive evaluation is observed in the development of 11 out of the 13 considered competences. Where, cross-disciplinary competences, averaged 3.35 points and professional ones 3.71 points out of a total of 4.0 points, allowing inferring that although most of the competencies were well evaluated, attention should be paid to adaptation to change and to autonomy and personal initiative

Keywords: Professional Competence, Transversal competence, student interns, Employers, Professional Practice.

\footnotetext{
1 Universidad de La Serena.Amunategui S/N Fono: 2204519. E-mail: cblanco@userena.cl

${ }^{2}$ Universidad de La Serena. Amunategui S/N. Fono; 2204519. E-mail: pcortes@userena.cl

${ }^{3}$ Universidad de La Serena. Amunategui S/N, Fono: 2204519. E-mail: ahernandez@userena.cl
} 
Revista de Investigación Aplicada en Ciencias Empresariales 


\section{INTRODUCCIÓN.}

Tal como lo señala (Gálvez, 2013), la sociedad actual, marcada por la globalización y los cambios constantes, exige en las personas que se harán cargo de ella, actores proactivos, que sean capaces de responder de manera clara e inmediata a las diversas vicisitudes que en su interior se susciten, pues hoy en día, el conocimiento por sí solo no es suficiente, se debe ser capaz de optimizar este conocimiento mediante una adecuada gestión del mismo, donde los saberes conceptuales, procedimentales, y actitudinales, no deben ser vistos como una gama de información la cual simplemente debe ser internalizada, sino más bien, integrada entre si y significada por los individuos, transformándose en atributos personales que harán la diferencia entre quien posee el conocimiento y quien sabe manejarlo.

He allí, el nuevo desafío de la Educación Superior en el Chile actual, donde un ejemplo de esto, es lo llevado a cabo por la Carrera de Auditoría de la Universidad de La Serena, carrera que a partir del año 2014 se ha sometido a un proceso de renovación y transformación curricular, transitando de un Modelo Pedagógico centrado en los objetivos a un modelo centrado en el desarrollo de competencias, donde un aspecto importante a considerar después de 4 años se haberse implementado este nuevo modelo, está dado por evaluar por parte de los empleadores, qué tan desarrolladas se encuentran las competencias tanto profesionales como transverales declaradas en el perfil de egreso de esta carrera, focalizando su centro de atención, en los estudiantes que realizaron su práctica profesional durante el año 2017. De allí que se desprendan los siguientes objetivos de investigación:

\section{OBJETIVO GENERAL.}

Evaluar por parte de los empleadores el desarrollo de competencias profesionales y transversales presentes el perfil de egreso y requeridas en la Práctica Profesional en los estudiantes de la Carrera de Auditoría de la Universidad de La Serena, cohorte 2014.

\section{Objetivos específicos.}

a) Identificar las competencias profesionales y transversales presentes en el perfil de egreso y requeridas en la Práctica Profesional de los estudiantes de la Carrera de Auditoría de la Universidad de La Serena cohorte 2014, a través de un análisis documental de la matriz de consistencia presentada por esta carrera.

b) Medir el desarrollo de las competencias profesionales y transversales presentes en el perfil de egreso y requeridas en la Práctica Profesional en los estudiantes de la Carrera de Auditoría cohorte 2014 de la Universidad de La Serena, mediante una encuesta de carácter cerrado y con indicadores tipo Likert realizada a los empleadores 
c) Jerarquizar las competencias profesionales y transversales presentes en el perfil de egreso y requeridas en la práctica profesional, acorde estas a su desarrollo en los estudiantes de la Carrera de Auditoría cohorte 2014 de la Universidad de La Serena, utilizando para esto un análisis estadístico dado por el establecimiento de puntuaciones promedios acorde a las respuestas entregadas en la encuesta realizada a los empleadores.

\section{METODOLOGÍA EMPLEADA.}

Enfoque de investigación: El enfoque utilizado, es el enfoque cuantitativo. Pues este usa la recolección de datos numéricos por medio de diferentes procedimientos estandarizados y el análisis de estos datos mediante procedimientos estadísticos, para así probar una hipótesis, lo cual se adecua perfectamente a los objetivos requeridos.

Diseño de investigación: Las investigaciones no experimentales buscan observar fenómenos en su contexto natural, sin la manipulación deliberada que se hace en las investigaciones experimentales.

En el caso de este estudio, se busca medir como las variables mencionadas (competencias), se encuentran desarrolladas y son puestas a disposición en la Práctica Profesional bajo un contexto natural, por lo tanto, no es necesario una manipulación de las variables.

Tipo de investigación: Se establece un tipo de investigación descriptiva, donde lo que se busca es medir el desarrollo de las competencias profesionales y transversales presentes en el perfil de egreso. De allí, que se utilice un análisis por frecuencia como base, y una análisis estadístico a través del establecimiento de medias a la hora de evaluar el desarrollo de estas competencias.

Alcance del estudio: El alcance de la investigación es del tipo transversal, ya que este diseño buscar estudiar los hechos en el aquí y ahora, acorde esto a su temporalidad.

\section{Técnicas de investigación:}

a) Encuesta: Técnica orientada a evaluar a los 25 estudiantes de la cohorte 2014 que realizaron su práctica profesional por parte de los 15 empleadores que recibieron a estos estudiantes considerando para esto un análisis por frecuencia, permitiendo así establecer tendencias.

b) Análisis estadístico: Focalizado en el establecimiento de la media muestral para cada variable considerada, permitiendo así, la jerarquización de las competencias evaluadas, donde a cada indicador seleccionado se le asigna un puntaje. Este puntaje va de $1 \mathrm{a}$ 4, dependiendo del indicador escogido. Donde 1 es asociado a estar en desacuerdo, 2 a estar 
medianamente de acuerdo, 3 a estar de acuerdo y 4 estar totalmente de acuerdo.

c) Análisis documental: Centrado en indagar en el estado del arte del tema central de la investigación que es llevada a cabo.

\section{MARCO TEÓRICO.}

\section{La innovación curricular y el desarrollo de competencias en las Universidades Chilenas.}

Autores como (Tobón, 2007) y (Gálvez, 2013), señalan que los modelos paradigmáticos que durante décadas primaron en la sociedad han comenzado a mostrar espacios vacíos, dando paso a una crisis paradigmática que denuncia los errores de una cosmovisión anterior, y enalteciendo el surgimiento de una nueva forma de entender la vida, cuya ramificación se extiende a cada una de las esferas de la sociedad, donde la educación no queda indiferente a estas transformaciones, enfrentándose ante una nueva panorámica, donde el modelo conductista comienza a dar paso a un nuevo modelo, focalizado en el estudiante y en su proceso de construcción del aprendizaje. Se está en presencia así del constructivismo.

Es así, que en el marco de un nuevo paradigma educativo propuesto a partir de los requerimientos de la sociedad del conocimiento y de la globalización, es la voz de los distintos actores la que da cuenta de la importancia de una visión centrada en la carga académica, los créditos académicos, la innovación, el papel activo del discente y el currículum basado en competencias,

Como parte de los cambios paradigmáticos que se han venido suscitando en los últimos años, las instituciones chilenas de Educación Superior que componen el sistema educativo actual, han ido reaccionando desde las más diversas y variadas formas. Pues producto de estos mismos cambios, surgen nuevos fenómenos, los cuales han llevado a estas instituciones a replantearse su forma de proceder, siendo la innovación curricular una de las principales preocupaciones de este sistema educativo, donde es el Consejo de Rectores de Universidades Chilenas (CRUCH), quien ha promovido y alentado las diversas formas en que esta innovación se ha presentado en dichas instituciones.

Es así que para el (CRUCH, 2011), la innovación curricular, sin descuidar la solvencia cognoscitiva y la competencia profesional, enfatiza la formación de individuos que adquieran un carácter reflexivo, metacognitivo, ético, crítico, innovador y adaptable. Buscando revertir la excesiva rigidez del proceso educativo y sus repercusiones. Sobre la base de este propósito, el conjunto de instituciones del $\mathrm{CRUCH}$, ha tomado decisiones sintonizadas con el contexto nacional e internacional, tendientes estas a explorar nuevas formas de enfrentar los procesos de enseñanza-aprendizaje, organizados en programas de grados y títulos, buscando incrementar así la calidad. 
Desde la perspectiva del (CRUCH, 2011), es la innovación curricular el camino adecuado para resolver los diversos problemas suscitados y lograr cumplir con los desafíos establecidos. Dicha innovación propende actualmente al desarrollo de competencias en función de un perfil de egreso, el cual a su vez, va cambiando de acuerdo a las necesidades de la sociedad, identificando competencias específicas y genéricas para cada nivel y asignatura, donde si bien el primer tipo de competencias señaladas adquieren gran importancia, dada las características propias que una profesión demanda, no puede dejarse de lado el segundo tipo de competencias enunciadas (transversales o genéricas), al ser estas la base para un desarrollo integral del ser humano. Sobre la base de esto, es que este organismo opte por seguir una formación que potencie ambos cuerpos de competencias, donde la evaluación del desarrollo de estas mismas, se ha de transformar en un aspecto fundamental a la hora de asegurar la concreción de este nuevo Modelo Educativo.

Es así, que a nivel nacional, se ha instaurado la evaluación de competencias tanto profesionales como transversales, como requisito fundamental esto a la hora de llevar a cabo un diagnóstico certero respecto a la formación del estudiantado y a la concordancia con lo declarado en los nuevos perfiles de egreso de las carreras. Algo enfatizado por instancias como es el caso de (Proyecto Tuning A.L, 2007).

\section{El surgimiento del concepto de competencias.}

Distintas son las definiciones que se plantean frente a este término, tal es el caso de autores como (Corominas, 2001), (Tobón, 2007) u organismos como el (CRUCH, 2010), aunque todos ellos concuerdan en definirlas como un conjunto de conocimientos, habilidades, destrezas, valores y actitudes, con un compromiso ético, que son desarrolladas por el ser humano y que facilitan su manejo óptimo en la sociedad, transformándose en atributos personales que le permiten al individuo enfrentar el sistema social con capacidad de reto y bajo una mirada crítica y resolutoria.

(Tobón, 2007), define las competencias como:

Procesos complejos de desempeño con idoneidad en determinados contextos, integrando diferentes saberes (saber ser, hacer, conocer y convivir), para realizar actividades y/0 resolver problemas con sentido de reto, motivación, flexibilidad, creatividad, comprensión y emprendimiento, dentro de una perspectiva de procesamiento metacognitivo, mejoramiento continuo y compromiso ético, con la meta de contribuir al desarrollo personal, a la construcción y afianzamiento del tejido social, a la búsqueda del desarrollo económico-empresarial sostenible. (p.17) 


\section{Las competencias profesionales como sello distintivo de las profesiones.}

Dentro del ámbito de las competencias, una tipología de estas, está dada por las que se conocen como competencias profesionales, las cuales suelen ser llamadas también competencias técnicas o verticales, y hacen referencia a aquel conjunto de conocimientos, habilidades y destrezas, adquiridas por el individuo después de haber realizado una formación especializada. Cabe mencionar que la (OIT, 2012), ha definido el concepto de competencia profesional como: "La idoneidad para realizar una tarea o desempeñar un puesto de trabajo eficazmente y por poseer las calificaciones requeridas para ello, donde los conceptos de competencia y calificación, se asocian fuertemente" (p.1).

Es de esta manera, que es posible concebir las competencias profesionales, como una compleja estructura de saberes, necesarias para el desempeño de situaciones específicas de una profesión, donde se da una compleja combinación de conocimientos, habilidades y tareas, que se tienen que desempeñar en determinadas situaciones. Bajo una mirada similar al concepto entregado por la OIT, el (Proyecto Tuning A.L, 2007), establece: "Las competencias profesionales, son aquellas que se relacionan con cada área temática, y tienen una gran importancia para cualquier titulación, pues estas, están relacionadas con el conocimiento concreto de un área específica" (p.71).

Como se puede apreciar, el desarrollo de este tipo de competencias, exige al individuo no solo manejar los conocimientos propios de su área disciplinar, sino además saber el cómo y cuándo utilizarlos, actuando como una mirada resolutiva más que meramente academicista. Es así, que es posible aseverar, que las competencias profesionales son un elemento relevante no solo a la hora de construir mallas curriculares, sino también, al momento de contribuir en el desarrollo profesional de una persona y su desenvolvimiento en la sociedad.

\section{Competencias transversales en el marco del paradigma actual.}

Complementaria a las competencias verticales, surgen también las competencias transversales. Las cuales pueden ser definidas como aquel conjunto de conocimientos, habilidades, destrezas, actitudes y valores, de carácter genérico, necesarias para cualquier graduado universitario y que sustentan su accionar en la conjunción de saberes orientados a servir de base para el desarrollo de las demás competencias. Según el (Proyecto Tuning A.L, 2007), estas pueden ser definidas como: "aquellas competencias de base, que identifican los elementos compartidos y comunes a cualquier titulación. Estas a su vez, se complementan con las competencias relacionadas con una especialidad" (p.37).

Es así, que las competencias transversales, parecen emerger como respuesta a una sociedad cambiante, adquiriendo importancia a la hora de ayudar a resolver las problemáticas del sistema social, ya sea como capacidades a aplicarse en la vida laboral y social, como también como facilitadores de competencias de mayor especificidad. 
Es tal la importancia de estas competencias, que el (Proyecto Tuning A.L, 2007), ha definido el siguiente listado de competencias transversales para ser aplicado en los programas de titulación de América Latina:

\section{TABLA Nº1 LISTA DE COMPETENCIAS TRANSVERSALES PROYECTO TUNING}

A.L

\begin{tabular}{|l|l|}
\hline 1.- Capacidad de abstracción, análisis y sintesis. & 14.- Capacidad creativa. \\
\hline 2.- Capacidad de aplicar los conocimientos. & $15 .-$ Capacidad para resolver problemas. \\
\hline 3.- Capacidad para organizar y planificar el tiempo. & 16.- Capacidad para tomar decisiones. \\
\hline 4.- Conocimientos sobre estudio y profesión. & 17.- Capacidad para trabajo en equipo. \\
\hline 5.- Responsabilidad social y compromiso & 18.- Habilidades interpersonales. \\
\hline 6.- Capacidad de comunicación oral y escrita. & 19.- Motivar y conducir metas comunes. \\
\hline 7.- Capacidad de comunicación en idioma. & 20.- Preservación del medioambiente. \\
\hline 8.- Habilidades en el uso de TICs. & 21.- Compromiso con medio socio cultural. \\
\hline 9.- Capacidad de investigación. & 22.- Valor y respeto por la diversidad. \\
\hline 10.- Aprender y actualizarse permanentemente. & 23.- Trabajar en contextos internacionales. \\
\hline 11.- Buscar, procesar y analizar información. & 24.- Trabajar en forma autónoma. \\
\hline 12.- Capacidad critica y autocrítica. & 25.- Formular y gestionar proyectos. \\
\hline 13.- Capacidad para actuar en nueva situaciones. & 26.- Compromiso ético. \\
\hline & 27.- Compromiso con la calidad. \\
\hline
\end{tabular}

Fuente: Proyecto Tuning A.L (2007).

En función de esto mismo, el (Proyecto Tuning A.L, 2007), ha propuesto también el siguiente esquema que vincula las competencias transversales con las competencias profesionales:

\section{ESQUEMA N¹: VINCULACIÓN DE COMPETENCIAS TRANSVERSALES Y PROFESIONALES.}

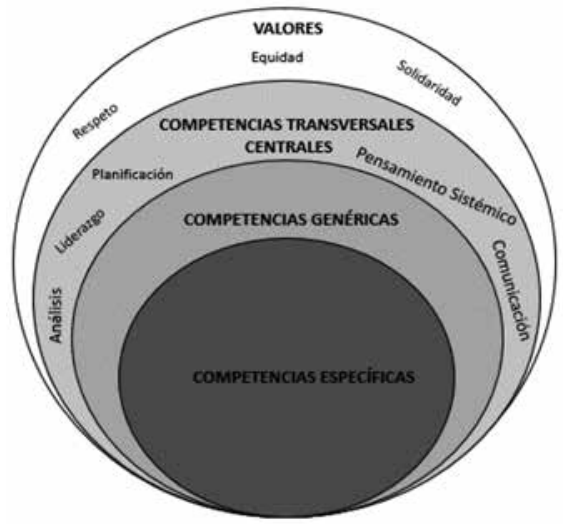

Fuente: Proyecto Tuning AL. (2007)

Como se puede apreciar en el esquema anterior, para propiciar el desarrollo de competencias, es necesario potenciar en primer lugar valores, valores que le confieran a la persona una aptitud determinada a la hora de enfrentar el conocimiento, permitiéndole así desarrollar saberes bases, conocidos estos como competencias transversales centrales y con esto competencias genéricas, lo que en la medida que sean potenciadas, sientan los pilares para el desarrollo de saberes más específicos y con un mayor nivel de abstracción de conocimiento. 


\section{RESULTADOS.}

Para lograr los objetivos de investigación propuestos, fueron diversas las técnicas investigativas llevadas a cabo, donde producto de estas, es posible establecer los siguientes resultados:

\section{Respecto de las Competencias profesionales y transversales presentes en el perfil de egreso y requeridas en la Práctica Profesional.}

Mediante un análisis documental realizado, es posible precisar que La Carrera de Auditoría de la Universidad de La Serena, a partir del año 2014 se somete a un proceso de renovación curricular, respondiendo de esta manera a las exigencias de un nuevo Modelo Educativo, modelo centrado en la formación en competencias, donde producto de esto mismo, se generan cambios considerables en dicha carrera y en sus programas de clases, elaborándose así una matriz de consistencia sobre la base de su perfil de egreso, y por ende, de las competencias tanto profesionales como transversales declaradas en este.

De esta manera, y recurriendo a la matriz de consistencia del perfil de egreso de esta carrera, es que posible establecer las siguientes competencias tanto profesionales como transversales, como se puede apreciar en la Tabla №2, que deberán ser desarrolladas y evidenciadas en la Práctica profesional, como se puede apreciar en la tabla №2:

\section{Tabla N2: Competencias profesionales y transversales en la Carrera de Auditoría.}

\section{PERFIL DE EGRESO.}

Se busca la formación de un profesional que sea capaz de: a).- Evaluar estados financieros de acuerdo a los principios y normas de contabilidad, b).- diseñar sistemas de información donde se apliquen adecuadamente las teorías contables y administrativas, el registro de operaciones y se respeten las normas, c).- determinar la carga impositiva de las empresas y personas respetando la legislación tributaria vigente, d).- desarrollar auditorías externas, planificando y ejecutando auditorías financieras, tributarias y de gestión, e).- desarrollar auditorías internas y f).- participar en la dirección y gestión de unidades estratégicas.

Complementario a lo anterior, ha declarado también, una serie de aptitudes deseables que atraviesan su plan de estudio, y se transforman en los pilares transversales que sustentan su formación académica. Es así que se evidencia la importancia de: a).- desarrollar la capacidad de lectura comprensiva, b).- desarrollar la adaptación al cambio, c).- desarrollar la capacidad para el estudio de disciplinas numéricas y aplicaciones lógicas, d).- capacidad para trabajar en equipo, independencia y liderazgo.

\begin{tabular}{|l|l|}
\hline \multicolumn{1}{|c|}{ Competencias Profesionales } & Competencias Transversales. \\
\hline $\begin{array}{l}\text { 1.- Distinción de los procesos económicos que se generan en } \\
\text { las empresas y los criterios de registro contable. }\end{array}$ & 1.- Responsabilidad \\
\hline $\begin{array}{l}\text { 2.- Aplicación de las normas de contabilidad y participación en } \\
\text { los procesos de generación de informes. }\end{array}$ & 2.- Trabajo en equipo \\
\hline $\begin{array}{l}\text { 3.- Valoración de la información financiera en la empresa y su } \\
\text { utilidad en la toma de decisiones. }\end{array}$ & $\begin{array}{l}\text { 3.- Autonomía e iniciativa } \\
\text { personal }\end{array}$ \\
\hline
\end{tabular}




\begin{tabular}{|l|l|}
\hline \multicolumn{1}{|c|}{ Competencias Profesionales } & \multicolumn{1}{c|}{ Competencias Transversales. } \\
\hline $\begin{array}{l}\text { 4.- Aplicación adecuada de las leyes, reglamentos y } \\
\text { disposiciones tributarias en los trabajos asignados. }\end{array}$ & 4.- Adaptación al cambio \\
\hline $\begin{array}{l}\text { 5.- Contribuye en los procesos de Auditoría y aplica los } \\
\text { procedimientos necesarios para su ejecución. }\end{array}$ & 5.- Compromiso ético. \\
\hline 6.- Aplicación satisfactoria de los conocimientos financieros & \\
\hline 7.- Valoración de la estructura orgánica de empresa. & \\
\hline $\begin{array}{l}\text { 8.- Incorporación herramientas tecnológicas y utilización de } \\
\text { manera eficiente para el desempeño de su trabajo. }\end{array}$ & \\
\hline
\end{tabular}

Fuente: Perfil de egreso Carrera de Auditoría.

2. Respecto de la evaluación por parte de los empleadores del desarrollo de las competencias profesionales y transversales en los alumnos de la Carrera de Auditoría cohorte 2014.

A la hora de medir el desarrollo de las competencias tanto profesionales como transversales en los 25 alumnos de VIII nivel cohorte 2014 que se encontraban cursando su Práctica Profesional durante el año 2017, se procedió a realizar una encuesta a los empleadores, pudiendo obtenerse de esta evaluación la siguiente información:

a) Competencias transversales en los alumnos de Auditoría.

- Competencia transversal evaluada: Responsabilidad.

\section{Gráfico No1: Demuestra responsabilidad en términos de: Cumplimiento de horarios, asistencia y otras obligaciones comprometidas.}

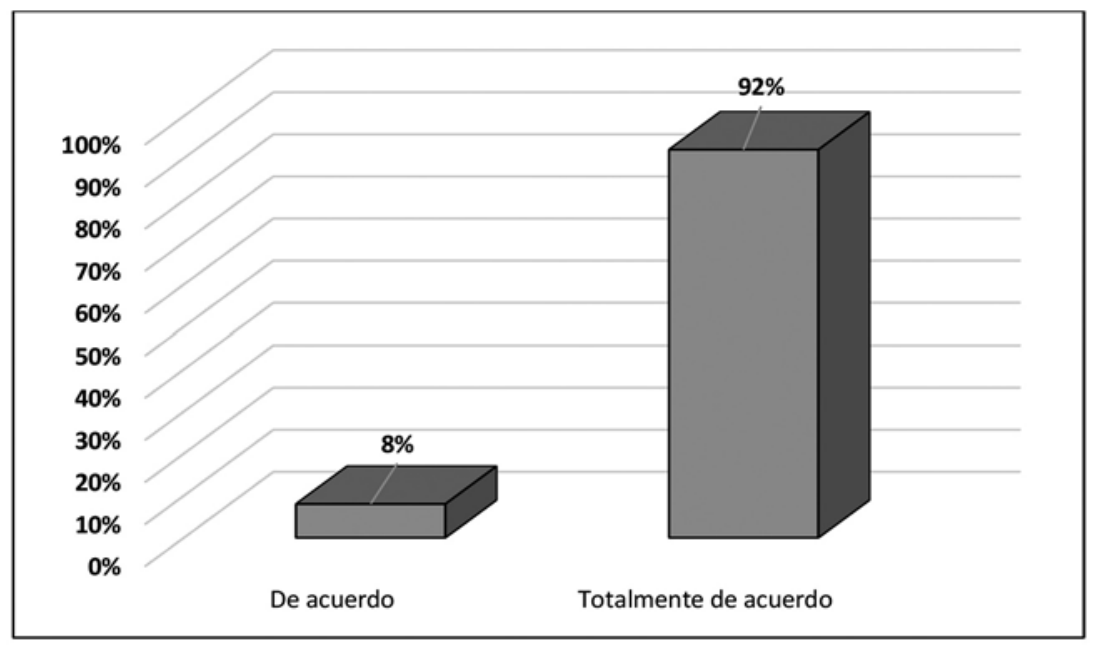

Fuente: Base de Práctica Carrera de Auditoría. 
Frente a la afirmación, demuestra responsabilidad en términos de cumplimiento de horarios, asistencia y otras obligaciones comprometidas, es posible señalar, según se aprecia en el Gráfico $\mathrm{N}^{0} 1$, que el $92 \%$ de los estudiantes, son evaluados de manera totalmente positiva por parte de los empleadores frente al desarrollo de esta variable, mientras un $8 \%$ de los discentes, son evaluados en términos aceptables.

Es importante mencionar también, que esta variable obtiene una media de 3,92 puntos de un total de 4,0 puntos, transformándola así, en una de las medias más altas obtenidas. Dando cuenta de esta manera, de alumnos que muestran una óptima adecuación a las normas y reglas establecidas en sus centros de Práctica Profesional.

- Competencia evaluada: Trabajo en equipo.

\section{Gráfico №2: Participa en los diálogos y discusiones del equipo desde sus ideas propias $\mathrm{y} / \mathrm{0}$ experiencias.}

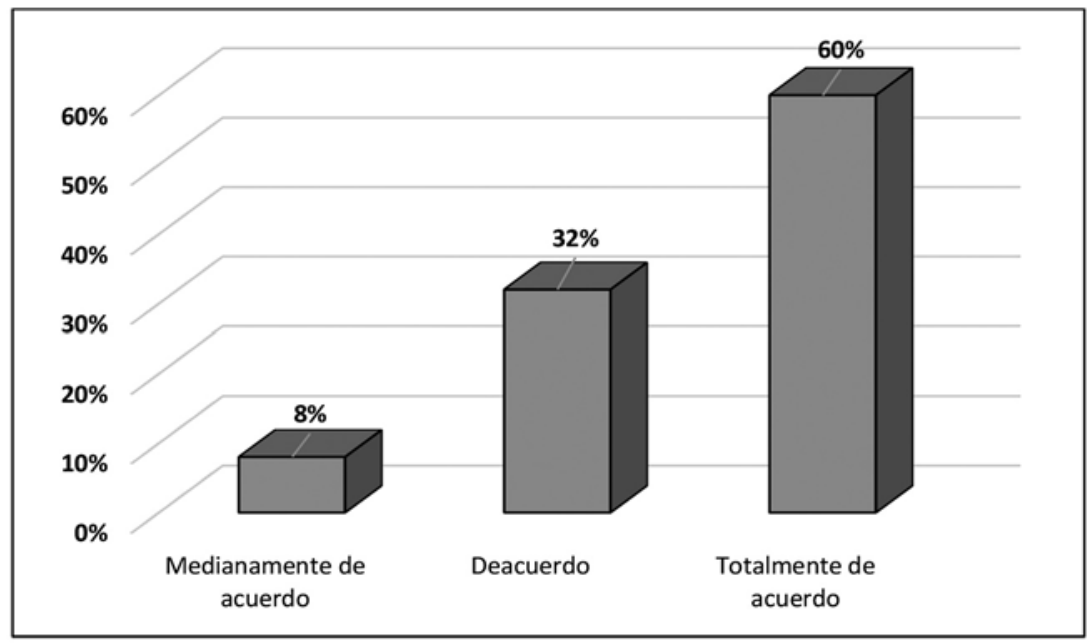

Fuente: Base de Práctica Carrera de Auditoría.

Con respecto a la aseveración, participa en los diálogos y discusiones del equipo desde sus ideas propias y/0 experiencias, es posible percatarse, que mientras el $60 \%$ de los estudiantes, son evaluados de manera totalmente óptima frente al desarrollo de esta variable por parte de los empleadores, un $32 \%$ de los alumnos, son evaluados en términos óptimos, tal como se muestra en el gráfico N02.

Llama la atención en esta variable, la aparición del indicador medianamente de acuerdo, donde un $8 \%$ de los alumnos, son 
evaluados en términos medios (medianamente de acuerdo), lo cual si bien no afecta la percepción óptima de lo evaluado, si da cuenta de un aspecto que debe ser considerado, más aun tomando en cuenta, que el participar activamente en los diálogos y discusiones, es uno de los principales atributos que debe poseer el Contador Público Auditor, ya que este profesional, trabaja directamente con equipos tanto multidisciplinarios como transdiciplinarios.

Respecto a la media obtenida de esta variable, esta promedia 3,52 puntos de un puntaje óptimo de 4,0 puntos, lo cual si bien se considera adecuado, esta no se encuentra entre las principales mayorías obtenidas en este estudio.

Corolario a lo anterior, otra variable relacionada con la competencia de trabajo en equipo, guarda relación con el involucramiento de los alumnos ante situaciones relevantes, pudiendo obtenerse al evaluar esta variable, el siguiente gráfico:

\section{Gráfico N³: Se involucra cuando surge una situación relevante.}

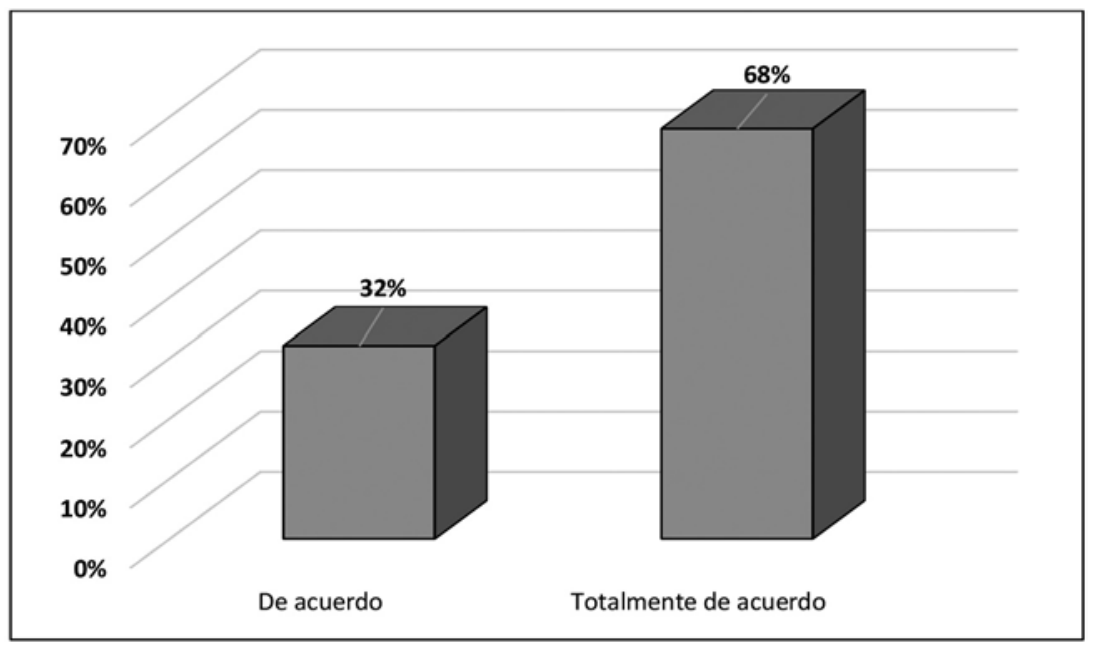

Fuente: Base de Práctica Carrera de Auditoría.

Como se puede apreciar en el Gráfico №3, la mayoría de los estudiantes, son evaluados en términos totalmente positivos por parte de los empleadores frente a este tópico, esto en un $68 \%$ de los casos, seguido de un $32 \%$ de los alumnos, que son evaluados en términos positivos, dando cuenta así, de estar de acuerdo con esta afirmación.

Respecto a la media obtenida frente a esta variable, ésta promedia 3,68 puntos de un total de 4,0 puntos, lo cual reafirma una 
participación activa de los estudiantes a la hora de formar parte de la resolución de problemas que se plantean en el grupo.

En resumen, y tomando en cuenta las dos variables consideradas en función de esta competencia, es posible señalar, una evaluación positiva del desarrollo esta, destacándose el compromiso del estudiante como parte ejecutora en la resolución de problemas, por sobre la del de generador de ideas que orienten la resolución del mismo, lo cual puede responder perfectamente a su calidad de alumno en práctica.

- Competencia transversal: Autonomía e iniciativa personal.

\section{Gráfico №4: Aporta información relevante y atingente a los desafíos que debe enfrentar en equipo.}

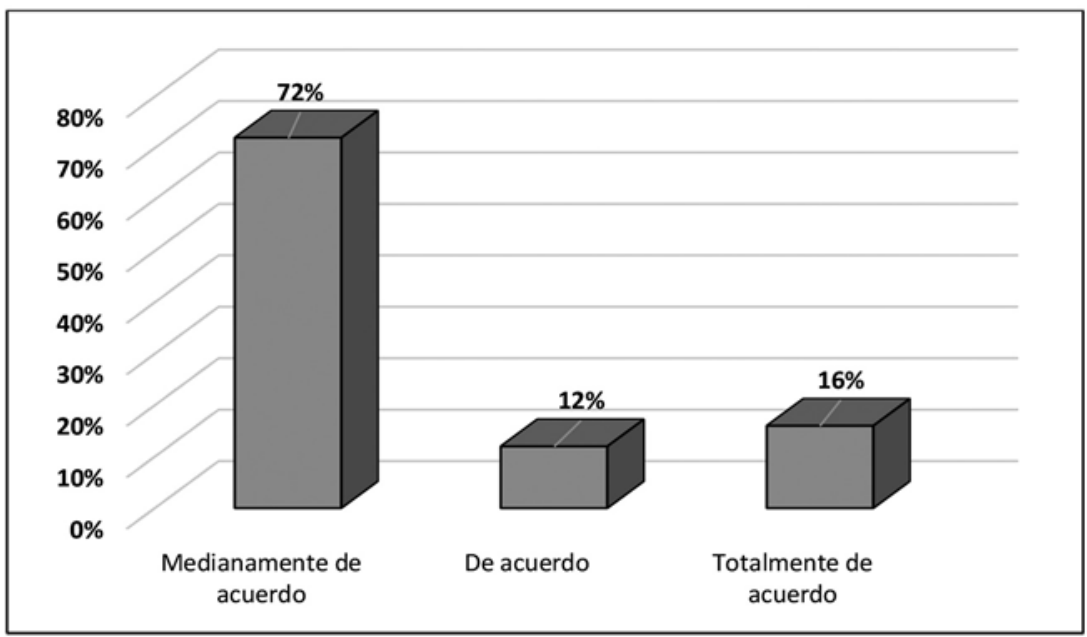

Fuente: Base de Práctica Carrera de Auditoría.

Como se puede evidenciar en el Gráfico No 4 , frente al postulado aporta información relevante y atingente a los desafíos que debe enfrentar el equipo, apuntando con esto a la proactividad e iniciativa que toma el alumno al interior del grupo, se puede señalar, que mientras un $72 \%$ de los discentes, son evaluados en términos medios por parte de los empleadores frente a esta variable, solo un $16 \%$ de ellos, son evaluados en términos totalmente positivos, y un $12 \%$, en términos positivos.

Como se puede apreciar, si bien la evaluación no es negativa, es imposible no percatarse, que la tendencia se inclina por una evaluación media respecto a este tópico.

Lo anterior, se ve reafirmado con la media obtenida en esta variable, la cual da cuenta de un puntaje de 2,44 puntos de un total de 4,0 puntos. 
En síntesis, el desarrollo de la competencia de autonomía e iniciativa personal, es evaluada en términos medios (medianamente lograda), siendo la que muestra el más bajo puntaje al compararlas con las otras competencias transversales precisadas en el estudio.

- Competencia de Adaptación al cambio.

Gráfico N5: Muestra ideas claras y determinantes frente al cambio.

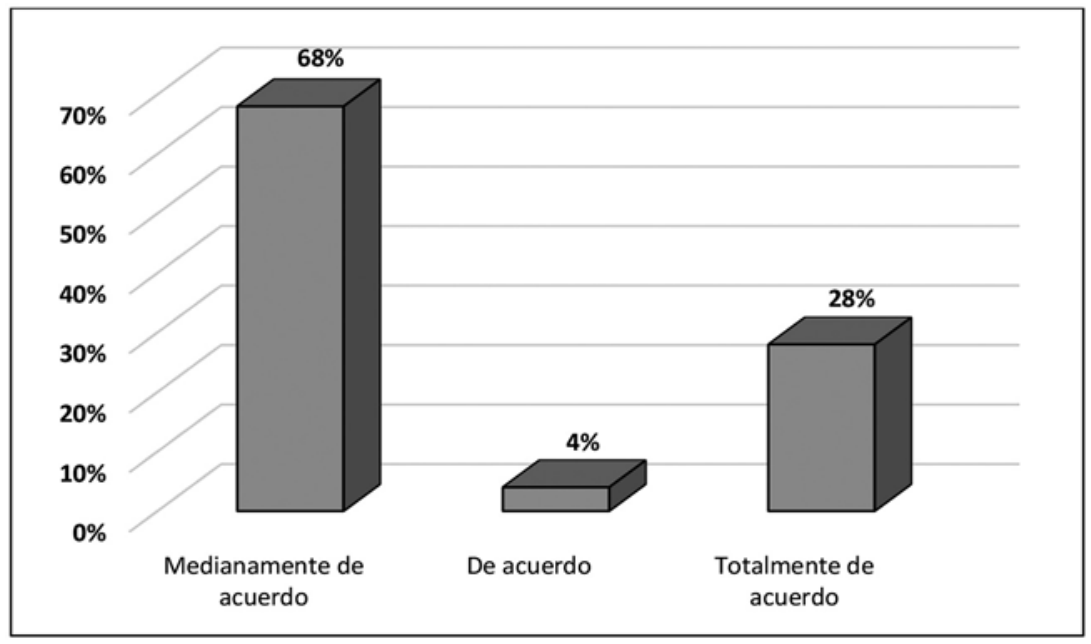

Fuente: Base de Práctica Carrera de Auditoría.

Ante la afirmación, que se presentan en el Gráfico №5 muestra ideas claras y determinante frente al cambio, es posible precisar, que mientras el $68 \%$ de los alumnos, son evaluados en términos medios por parte de los empleadores ante esta aseveración, un 28\% de los estudiantes son evaluados en términos totalmente positivos, y un $4 \%$ de ellos, en términos óptimos.

Lo anterior permite evidenciar una evaluación media (como medianamente lograda) de esta variable, obteniendo un promedio de 2,6 puntos de un total de 4,0 puntos.

A través de esto, puede aseverarse, que existe un desarrollo medianamente aceptable de la competencia de adaptación al cambio.

- Competencia transversal de la ética. 
Gráfico Nº: Muestra compromiso ético para con su disciplina y para con el entorno en el cual trabaja.

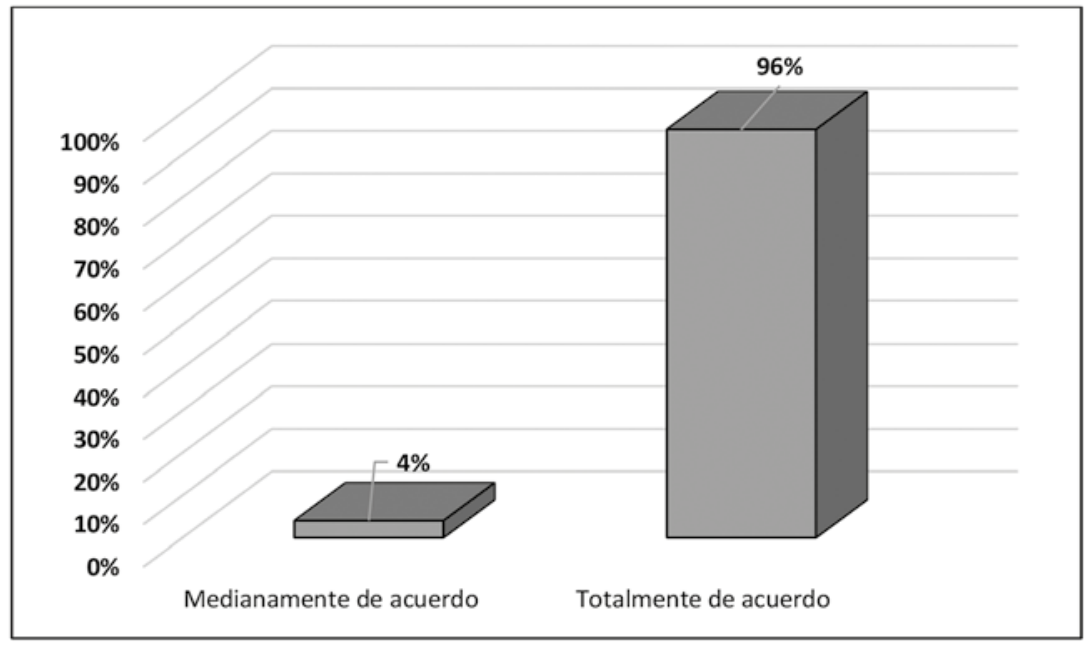

Fuente: Elaboración propia.

El Gráfico No6, da cuenta que frente a la variable, muestra un compromiso ético con su disciplina y para con el entorno en el cual trabaja, un $96 \%$ de los estudiantes, son evaluados por parte de los empleadores de manera totalmente satisfactoria, mostrando así, un alto desarrollo de la competencia de la ética en su Práctica Profesional, donde solo el $4 \%$ del total de discentes, son evaluados en términos óptimos, lo cual igualmente es positivo.

Esta evaluación, se ve reflejada también en la media obtenida, la cual es de 3,92 puntos de un total de 4,0 puntos, alcanzando así, la evaluación positiva más alta de las variables medidas orientadas a la evaluación del desarrollo de competencias de índole transversal, y quedando de manifiesto, la significación de los principios éticos entregados en la Carrera de Auditoría de la Universidad de La Serena a sus estudiantes.

b) Competencias profesionales en los alumnos de Auditoría.

- Competencia profesional: Distinción de los procesos económicos que se generan en las empresas y de los criterios de registro contable. 


\section{Gráfico N7: Distingue los procesos económicos que se generan en las empresas (instituciones) y los criterios de registro contable.}

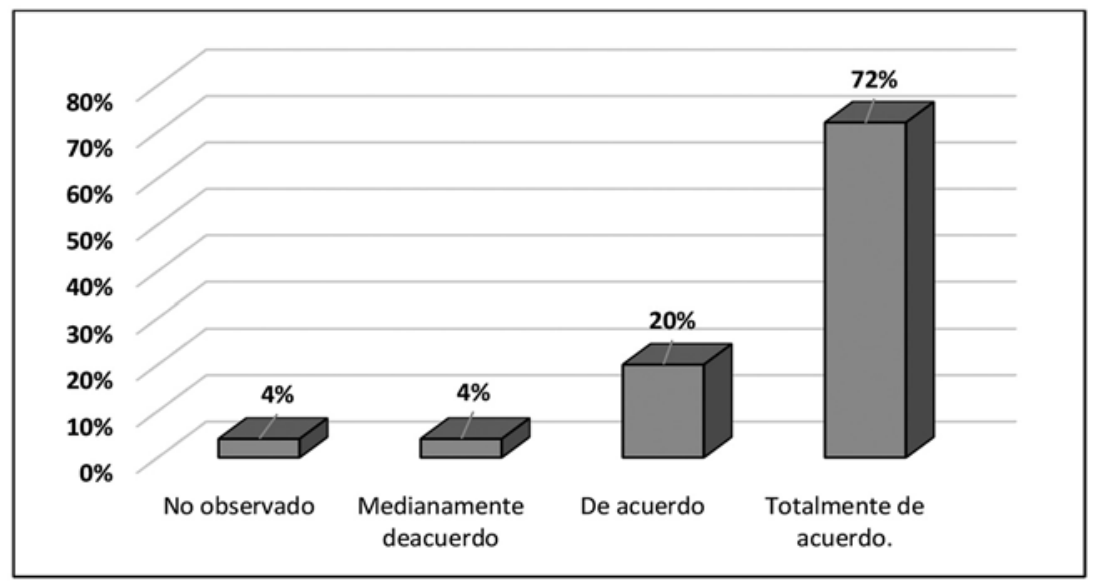

Fuente: Base de Práctica Carrera de Auditoría.

Como se puede apreciar en el Gráfico №7, frente a la afirmación, distingue los procesos económicos que se generan en las empresas y los criterios de registro contable, se puede señalar, que mientras un $72 \%$ de los estudiantes, son evaluados de manera totalmente positiva por parte de los empleadores frente a esta aseveración, un $20 \%$ de ellos son evaluados de manera positiva y un $4 \%$ en términos medios. Lo anterior da cuenta, de una evaluación óptima de esta competencia, evidenciándose por parte de los alumnos, una alta capacidad a la hora de poder diagnosticar la situación contable de una empresa, teniendo como base para esto, la información emanada a través de sus estados de cuenta. Esta aseveración, queda reafirmada a su vez, por una media obtenida de 3,71 puntos de un total de 4,0 puntos.

Es importante hacer alusión, que pese a esta evaluación positiva, el hecho de que el $20 \%$ de los alumnos sean evaluados solo en términos aceptables y un $4 \%$ en términos medios, influye en la no obtención del puntaje total. Se hace hincapié además, que el $4 \%$ de alumnos que no son evaluados en función de esta variable, responde a que esta competencia no es solicitada acorde a los requerimientos del área donde los estudiantes desarrollaron su práctica profesional. Por ende, este porcentaje no influye en la media.

- Competencia profesional: Aplicación de las normas de contabilidad y participación en los procesos de generación de informes de contabilidad. 
Gráfico N8: Aplica las normas de contabilidad y participa en los procesos de generación de informes de contabilidad.

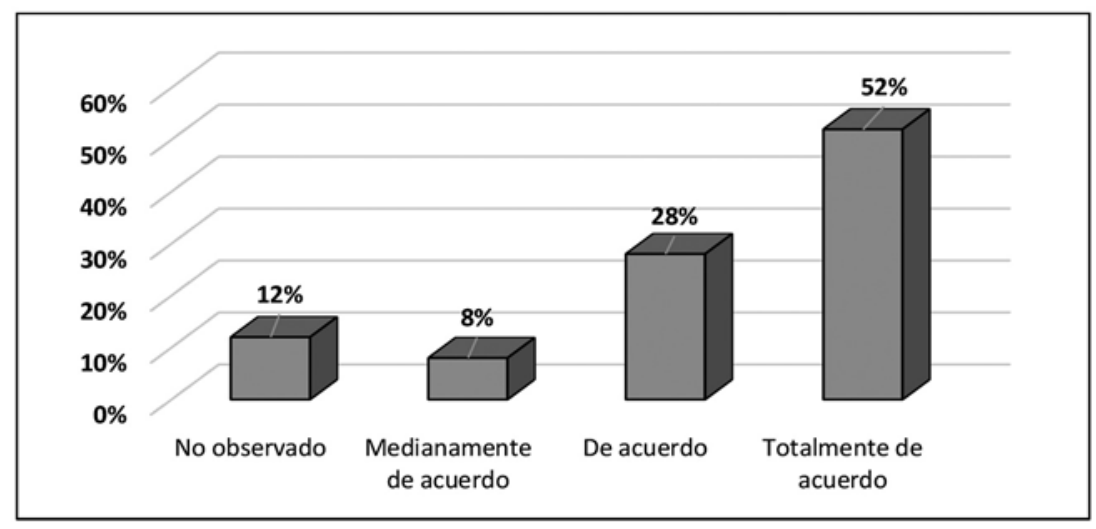

Fuente: Base de Práctica Carrera de Auditoría.

A través del Gráfico No8, se evidencia, que mientras un $52 \%$ de los alumnos, obtienen una evaluación totalmente positiva de esta variable por parte de los empleadores, un $28 \%$, obtiene una evaluación positiva y un $8 \%$, una evaluación media.

Para este caso, el porcentaje de alumnos que no fue evaluado en función de esta competencia, asciende a un $12 \%$.

A raíz de los datos anteriores, si bien es posible afirmar una evaluación positiva de esta variable (competencia), no deja de llamar la atención, el aumento de la valoración en términos medios, y la diminución de la evaluación totalmente satisfactoria (52\%), lo cual se ve reflejado en la media establecida en relación con esta competencia, la cual asciende a 3,50 puntos de un total de 4,0 puntos. Transformándola en la media más baja de las obtenidas en la evaluación de competencias profesionales, evidenciando la necesidad de potenciar aún más, tanto el rol activo del estudiante como la aplicación de los conocimientos contables en la elaboración de informes de contabilidad y en los procedimientos relacionados con esta área.

- Competencia profesional: Valoración del rol e importancia de la información financiera en la empresa y su utilidad en la toma de decisiones. 


\section{Gráfico $\mathrm{N}^{\circ}$ 9: Valora el rol e importancia de la información financiera en la empresa y su utilidad en la toma de decisiones.}

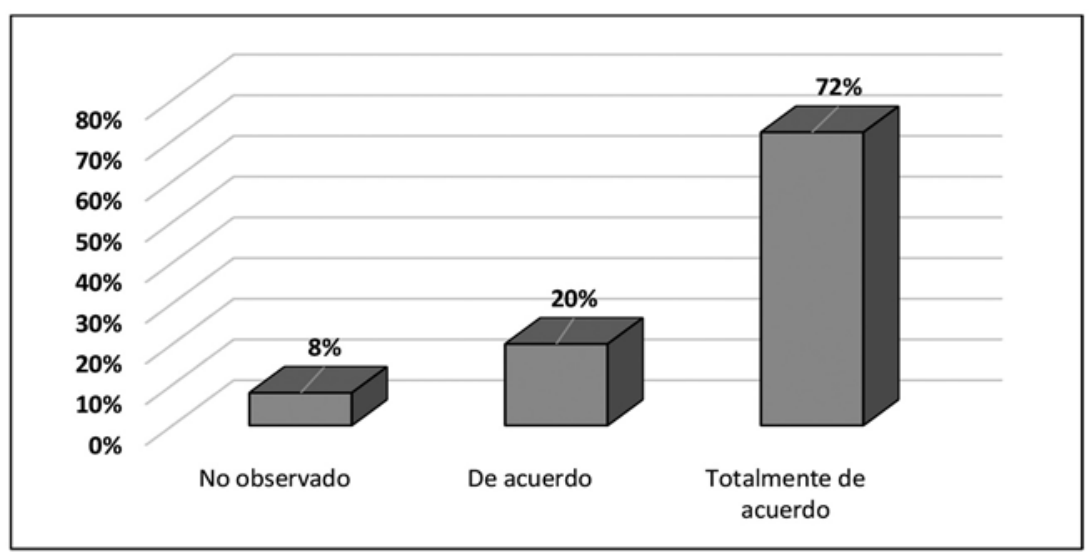

Fuente: Base de Práctica Carrera de Auditoría.

Por medio del Gráfico №9, es posible señalar, que el $72 \%$ de los alumnos que cursaron su Práctica Profesional, son evaluados de manera totalmente positiva respecto al desarrollo de esta competencia según los empleadores, seguido de un $20 \%$ que son evaluados en términos óptimos.

Lo anterior refleja entonces, una alta valoración que se le da a la información financiera y a la importancia que esta tiene en la toma de decisiones al interior de las organizaciones.

Esta tan alta valoración, se reafirma con la media obtenida en esta variable, la cual es de 3,78 puntos (de un total de 4,0 puntos), transformándola en una de las competencias profesionales como mejor evaluación.

Es importante señalar también, que el porcentaje de alumnos que no fueron evaluados en función de esta variable, es de un $8 \%$.

- Competencia profesional: Demuestra y aplica conocimientos financieros. 


\section{Gráfico №10: Demuestra y aplica conocimientos del área financiera en el desempeño de su práctica.}

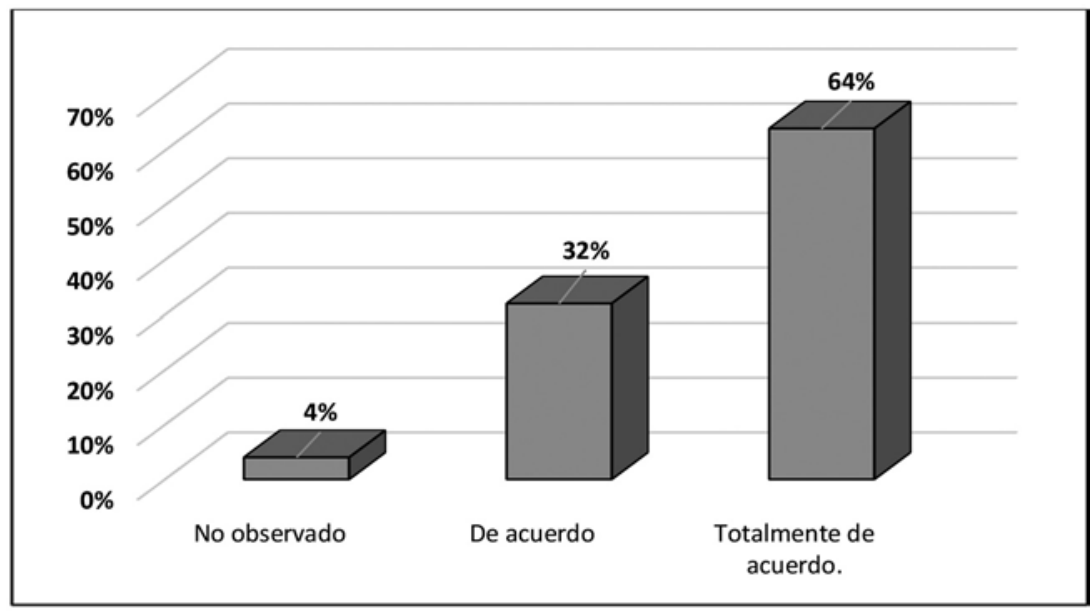

Fuente: Base de Práctica Carrera de Auditoría.

El Gráfico №10, da cuenta que el $64 \%$ de los alumnos, son evaluados de manera totalmente satisfactoria por parte de los empleadores frente al desarrollo de esta competencia, un $32 \%$ de ellos, son evaluados de manera satisfactoria y un $4 \%$, no son evaluados, debido a que esta competencia no fue requerida en el área desempeñada.

Es así, que los datos entregados, permiten afirmar una evaluación positiva del desarrollo de esta competencia, reafirmándose esto, con una media de 3,67 puntos de un total de 4,0 puntos, evidenciándose, un adecuado manejo de los conocimientos financieros en su aplicación.

- Competencia profesional: Aplicación adecuada de las leyes, reglamentos y disposiciones tributarias en los trabajos asignados. 
Gráfico №11: Aplica adecuadamente las leyes, reglamentos y disposiciones tributarias en los trabajos asignados.

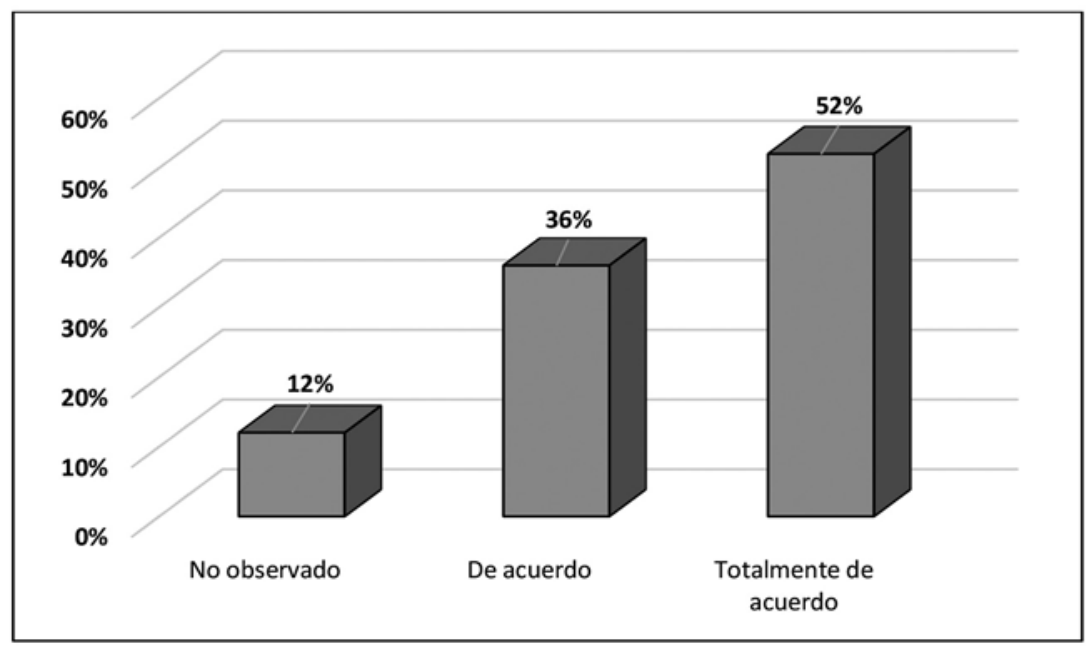

Fuente: Base de Práctica Carrera de Auditoría.

Producto del Gráfico N011, puede señalarse, que mientras el $52 \%$ de los alumnos que realizaron su Práctica profesional son evaluados de manera totalmente positiva frente a esta variable, un $36 \%$ ellos, son evaluados de manera positiva.

En el caso de los alumnos que no fueron evaluados en función de esta variable, el porcentaje asciende a un $12 \%$.

Como es posible apreciar, si bien la valoración de esta competencia es positiva, se observa un elevado porcentaje de alumnos que son evaluados en una categoría óptima pero no totalmente satisfactoria, lo que lleva a que la media obtenida en el desarrollo de esta competencia, alcance los 3,59 puntos de un total de 4,0 puntos, dejándola entre las competencias que ponderan los promedios más bajos entre los atributos profesionales evaluados. Lo anterior lleva a reafirmar, que pese a su valoración positiva, existen vacíos en la aplicación de conocimientos tributarios.

- Competencia profesional: Contribución en los procesos de auditoría y aplicación de los procedimientos necesarios para su ejecución. 


\section{Gráfico No12: Contribuye en los procesos de Auditoría y aplica los procedimientos necesarios para su ejecución.}

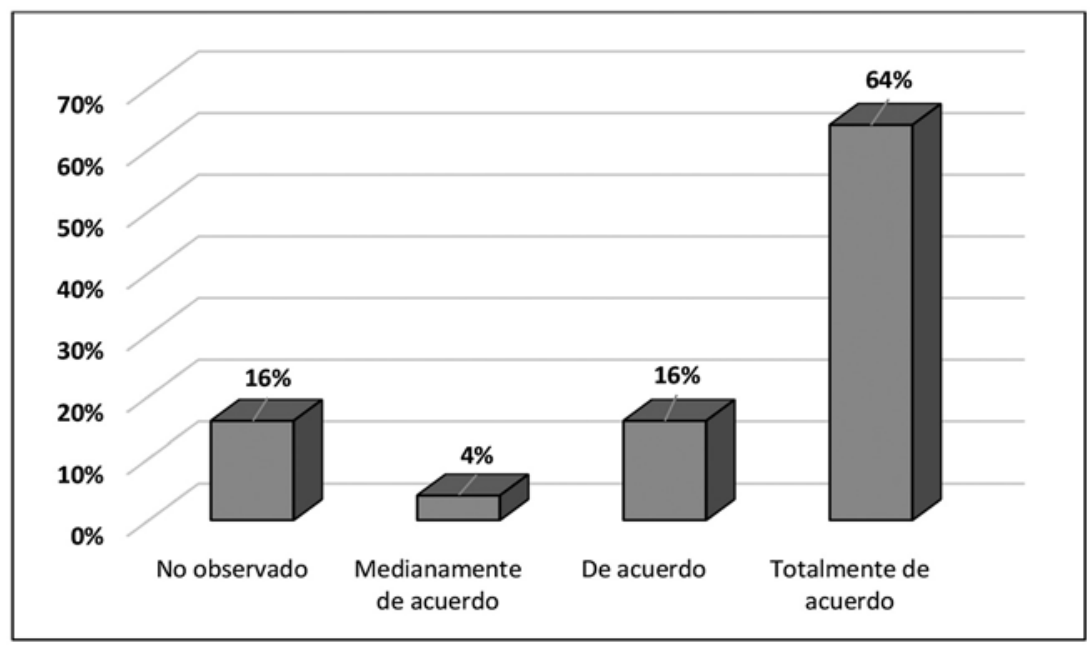

Fuente: Base de Práctica Carrera de Auditoría.

Del Gráfico N012 puede desprenderse, que un 64\% de los alumnos, son evaluados de manera totalmente positiva respecto al desarrollo de esta variable, seguido de un $16 \%$ de los estudiantes, que son evaluados de manera satisfactoria y un $4 \%$, que son evaluados en términos medios. Esto permite aseverar, una evaluación satisfactoria de esta competencia, concentrándose la tendencia en una evaluación altamente positiva, aunque visualizándose la aparición de una evaluación media en un $4 \%$ de los estudiantes, lo que sumado al $16 \%$ de alumnos, que son evaluados solo en términos satisfactorios, impide el establecimiento de una media más alta, la cual alcanza los 3,80 puntos de un total de 4,0 puntos. A pesar de esto, el desarrollo de esta competencia, se sitúa en una de las primeras tres variables profesionales mejor evaluadas.

- Competencia profesional. Valoración efectiva de la estructura orgánica de la empresa y demostración de compromiso con el cumplimiento de las normas y procedimientos de la organización. 


\section{Gráfico №13: Valoración de la estructura orgánica de la empresa y demostración de compromiso con el cumplimiento de las normas y procedimientos de la organización.}

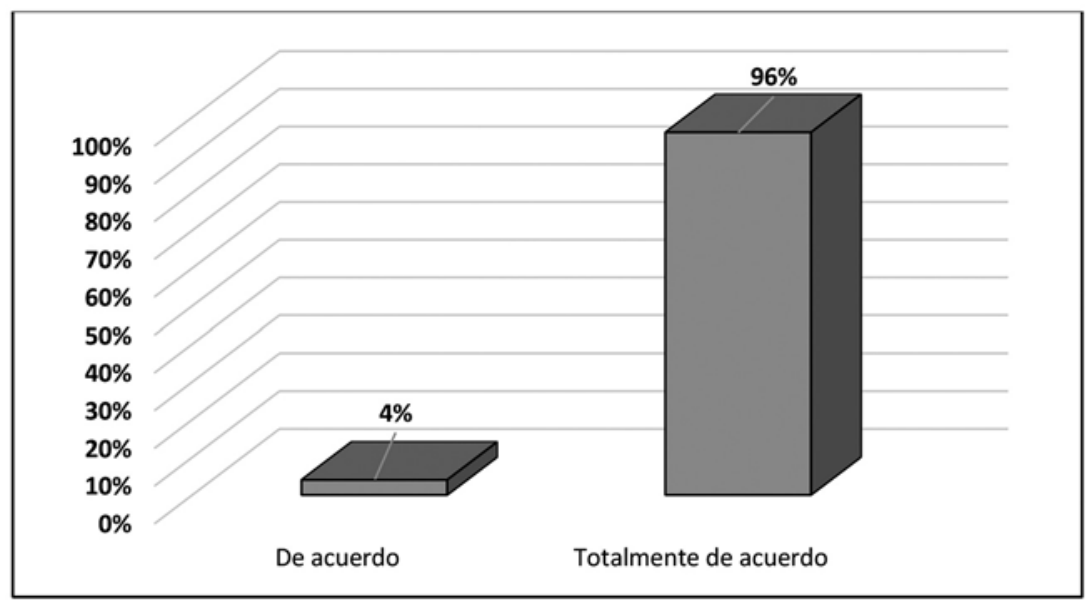

Fuente: Base de Práctica Carrera de Auditoría.

En el Gráfico №13 se permite visualizar, que mientras un $96 \%$ de Ios alumnos, son evaluados de manera totalmente óptima por parte de los empleadores frente a esta variable, solo un $4 \%$ de ellos, son evaluados de manera óptima, siendo ambos indicadores positivos, lo que da cuenta de un desarrollo alentador de esta competencia.

Es tal el grado de evaluación positiva de la misma, que su media asciende a 3,96 puntos de un total de 4,0 puntos, lo que da cuenta de que los alumnos cumplen satisfactoriamente con lograr respetar la orgánica de la empresa donde desempeñaron su práctica profesional, como también de las normas propias de la organización.

- Competencia profesional: Incorporación de herramientas tecnológicas y su utilización de manera eficiente para el desempeño de su trabajo. 
Gráfico №14: Incorpora herramientas tecnológicas y las utiliza de manera eficiente para el desempeño de su trabajo

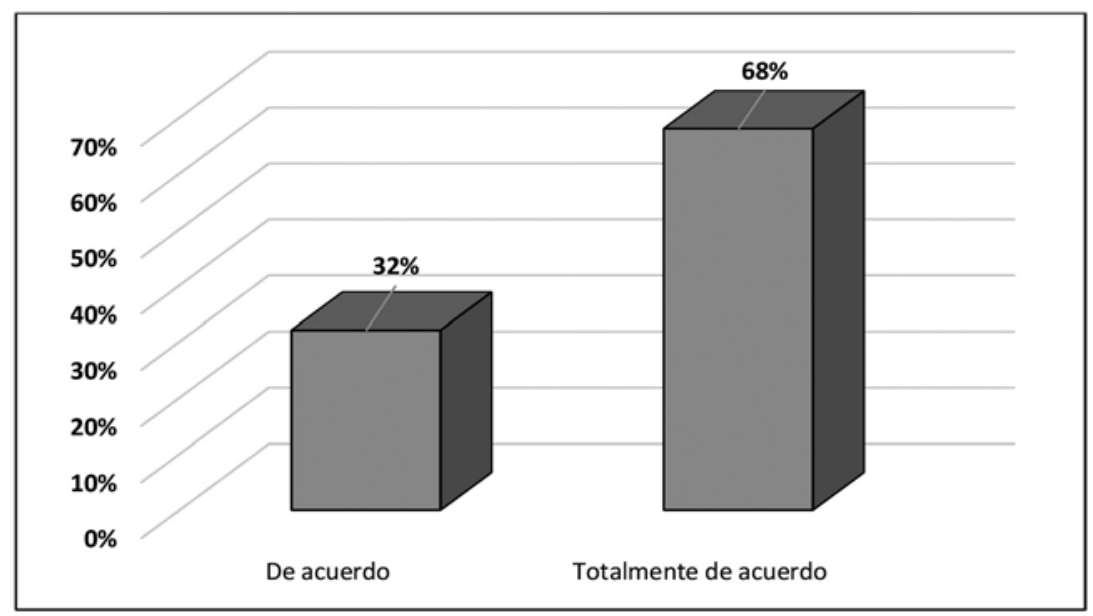

Fuente: Base de Práctica Carrera de Auditoría.

A través del Gráfico №14 se puede señalar, que mientras un 68\% de los alumnos que cursaron su Práctica Profesional son evaluados de manera totalmente satisfactoria frente al desarrollo de la variable considerada, un $32 \%$ de ellos, son evaluados de manera satisfactoria. Esto lleva a afirmar, un desarrollo óptimo de la competencia orientada a la utilización de herramientas tecnológicas propias de la profesión. Aunque es necesario hacer hincapié, que la media obtenida es relativamente baja en comparación con algunas de las otras competencias evaluadas, promediando 3,68 puntos de un total de 4,0 puntos.

\section{Respecto de la jerarquización del desarrollo de competencias profesionales y transversales en los alumnos de la Carrera de Auditoría cohorte 2014.}

Una vez medido el desarrollo de las competencias tanto profesionales como transversales en los alumnos de la Carrera de Auditoría que cursaron su Práctica Profesional, se procedió a comparar las medias obtenidas en cada competencia, esto con la finalidad de poder establecer una jerarquía que permitiera determinar cuáles son a juicio de los empleadores, aquellas competencias que se encuentra mejor desarrolladas en los estudiantes. Es así, que a continuación se presenta el siguiente esquema: 


\section{Gráfico Nº15: Media de competencias evaluadas en la Práctica Profesional.}

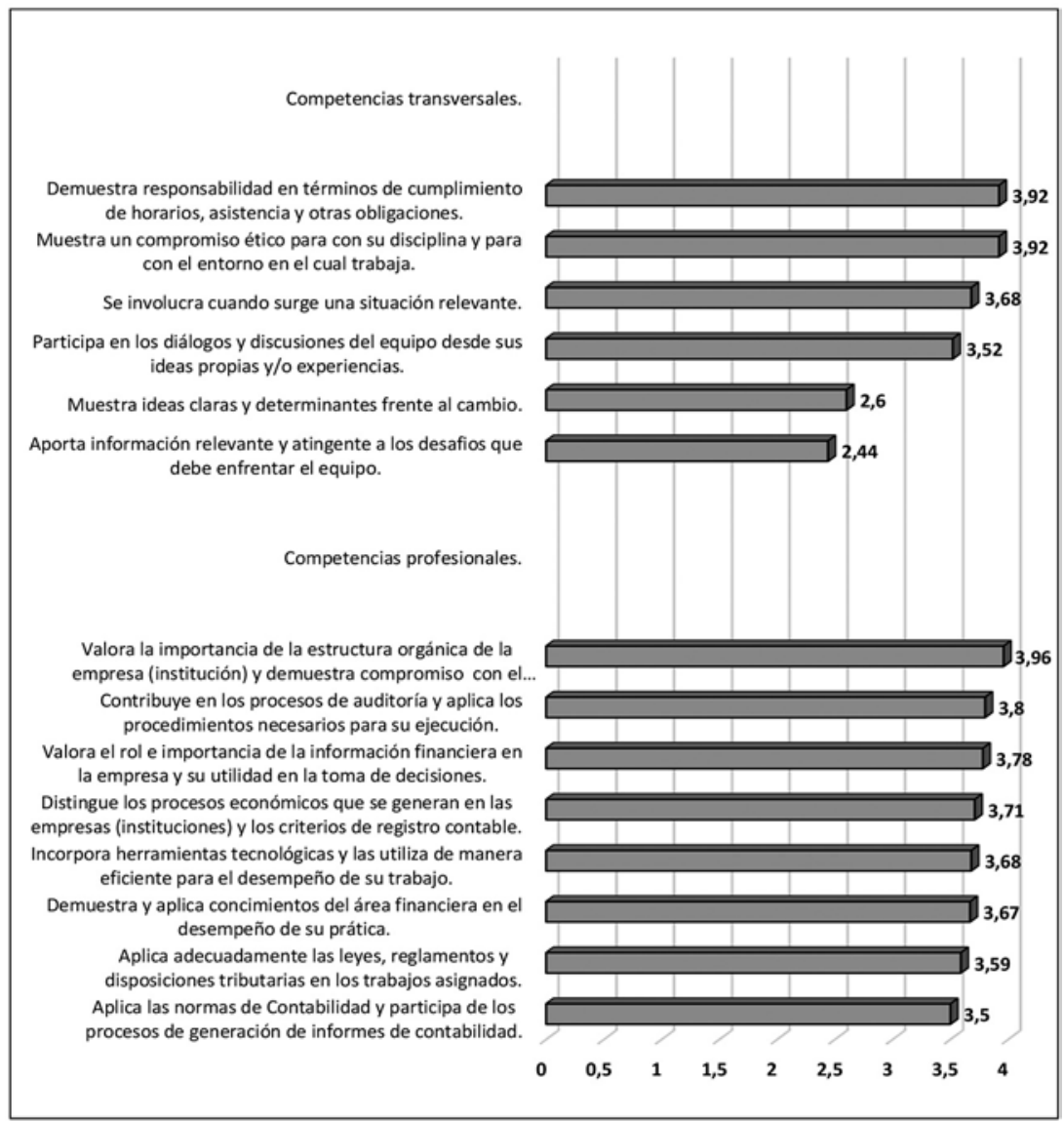

Fuente: Base de Práctica Carrera de Auditoría.

El Gráfico N015, permite visualizar dos grandes cuerpos de competencias, las cuales se encuentran divididas tanto en competencias transversales como profesionales.

Es así, que en el caso de las competencias transversales, es posible señalar la siguiente jerarquía respecto al desarrollo manifestado por estas en la Práctica Profesional:

Puesto №1: En un primer lugar, se encuentran tanto la competencia transversal de la ética con un promedio de 3,92 puntos de un total de 4,0 puntos, como la de la responsabilidad, con un mismo puntaje. Esto permite afirmar por medio de las variables medidas, que por una parte, los alumnos evaluados, demuestran un compromiso ético bastante marcado con el actuar bajo principios correctos en su desempeño profesional, considerando las áreas propias de su disciplina, así como en las relaciones e interacciones sociales que se establecen para con el entorno, y por otra, que tienen claramente significada la relevancia que adquiere el ser responsables con los compromisos adquiridos en las áreas laborales en las 
cuales se desempeñan, comprendiendo que no solo los conocimientos propios de su especialidad son suficientes, sino además, demostrando constantemente, un respeto por el lugar donde han realizado sus Prácticas Profesionales y por el personal que labora junto a ellos.

Puesto N²: En segundo lugar, es posible encontrar la competencia de trabajo en equipo, esta con una media de 3,68 puntos en la variable que guarda relación con el involucramiento ante situaciones relevantes, y con una media de 3,52 puntos, en la variable que guarda relación con la participación en los diálogos y discusiones al interior del grupo. Si bien esta última variable arroja una media menor que la anterior, a la hora de promediar ambas variables directamente relacionadas con el trabajo intergrupal, se obtiene una media de 3,60 puntos de un total de 4,0 puntos, permitiendo de esta manera, clasificar esta competencia en este lugar en función de su desarrollo.

Es así, que es posible afirmar, que los alumnos logran comprender satisfactoriamente la importancia que tiene el trabajo mancomunado a la hora de sortear los obstáculos que se les presentan.

Es necesario resaltar sí, que dentro de la competencia de trabajo en equipo, se muestra una mejor evaluación respecto al involucramiento ante situaciones relevantes, mostrando interés por participar en la resolución de las mismas como ente ejecutor, más que con el aportar con ideas propias en los diálogos y discusiones que se generan al interior del grupo, lo cual puede deberse al carácter de alumno practicante que el discente adquiere.

Puesto N³: En el tercer lugar, es posible encontrar la competencia de adaptación al cambio, la cual es traducida en la variable orientada a evaluar la capacidad de mostrar ideas claras y determinantes frente a situaciones imprevistas, promediando esta, una media de 2,60 puntos de un total de 4,0 puntos, lo que lleva a evidenciar una evaluación media de esta competencia, dando cuenta de que hay inconvenientes considerables a la hora de adaptase y asimilar las nuevas realidades que se hacen visibles.

Puesto $N^{\circ} 4$ : En el último lugar de esta jerarquización, se encuentra la competencia de autonomía e iniciativa personal, medida esta por la variable que guarda relación con el hecho de aportar con información relevante y atingente a los desafíos que debe enfrentar el equipo.

La media obtenida en esta variable es de 2,44 puntos de un total de 4,0 puntos, lo que lleva a reafirmar lo enunciado por los empleadores en los procesos de acreditación anteriores. Pues si bien los alumnos de auditoría muestran cierto desarrollo de esta competencia, aún hay aspectos que resolver, ya que existe en varios casos, cierta inclinación por actuar meramente en colectividad y no atreverse a actuar por cuenta propia.

A nivel global, es importante señalar, que el desarrollo de las competencias transversales, muestra una media de 3,35 puntos de un total de 4,0 puntos. 
Por otra parte, respecto al desarrollo de las competencias profesiones en los alumnos practicantes, propias estas de la formación profesional, es posible establecer la siguiente jerarquía:

Puesto No1: En primer lugar, se encuentra la valoración que los estudiantes le otorgan a la estructura orgánica de la empresa y a la demostración de su compromiso con el cumplimiento de las normas y procedimientos propios de la organización. Visualizándose de esta manera, un desarrollo óptimo por parte de estos alumnos, en función del sentido de pertenencia que estos generan con la gestión y administración en el plano estructural de la institución.

La media obtenida en esta variable, asciende a 3,96 puntos de un total de 4,0 puntos, transformándose así, en la competencia mejor evaluada respecto a su desarrollo. Demostrando con esto, que el alumno de Auditoría que cursa su Práctica Profesional, no solo está consciente de la relevancia que adquiere el adecuarse a los contextos y a los parámetros establecidos a la hora de contribuir eficientemente en las empresa donde desempeña su labor profesional, sino además, es capaz de plasmar este compromiso en la práctica.

Puesto $N^{\circ} 2$ : En este puesto, se encuentra una competencia profesional cuya media obtenida es de 3,80 puntos de un total de 4,0 puntos. Con esto se hace referencia a la competencia orientada a la contribución que los alumnos generan en los procesos propios de la Auditoría, concretándose esto en la aplicación de los procedimientos necesarios para su ejecución y su puesta en marcha.

De esta forma, se evidencia por parte de los estudiantes, un adecuado expertiz en esta dimensión, mostrando un óptimo desempeño en los procesos que guían el auditar, demostrando de esta manera, ser un aporte concreto a la hora mostrar la realidad visualizada al interior de una empresa.

Puesto N³: En este lugar, es posible encontrar aquella competencia orientada a la valoración del rol e importancia que los alumnos le asignan a la información financiera en la empresa y la utilidad que esta genera en la toma de decisiones, alcanzando este atributo profesional, una evaluación positiva en relación al desarrollo manifestado.

Lo anterior permite vislumbrar, que los alumnos de Auditoria que cursan su Práctica Profesional, están conscientes de que la información financiera es base a la hora de determinar la situación de la empresa y por ende de las medidas remediales que se han de considerar, transformándose así, en un insumo base para el Contador Público Auditor.

La media obtenida en esta competencia, asciende a 3,78 puntos de un total de 4,0 puntos, lo cual es considerado bastante alto, y la ubica en el tercer lugar de las competencias profesionales evaluadas en función de su desarrollo.

Puesto $N^{\circ} 4$ : En cuarto lugar, es posible encontrar la competencia focalizada en la capacidad de distinguir los procesos económicos que se generan en las empresas 
y los criterios de registro contable. Esta con una media de 3,71 puntos de un total de 4,0 puntos. Ubicándola en una escala más bien intermedia en relación con las demás competencias evaluadas.

El desarrollar adecuadamente esta competencia, muestra una comprensión adecuada en términos contables de los aspectos que deben ser considerados a la hora de evidenciar situación la cual atraviesa la organización, generándose de esta manera, la base para un diagnóstico certero de aspectos como los estados de cuenta de la misma.

Puesto $N^{\circ}$ : En quinto lugar, se hace presente la competencia orientada a la incorporación de herramientas tecnológicas y la utilización eficiente de estas para el desempeño de su trabajo.

Esta competencia, arroja una media de 3,68 puntos de un total de 4,0 puntos, dando cuenta de esta forma, si bien de una evaluación positiva de la misma respecto de su desarrollo, una ubicación en un rango medio, si la comparamos con aquellas evaluadas anteriormente.

El desarrollo óptimo de esta competencia en los alumnos de Auditoría que cursan su Práctica Profesional, hace alusión a un adecuado manejo de las tecnologías que están al servicio de la disciplina profesional, tal es el caso de software y programas computacionales.

Puesto $N^{\circ} 6$ : En el sexto lugar, es posible visualizar la competencia profesional orientada a la capacidad de demostrar y aplicar los conocimientos del área financiera en el desempeño de su práctica. El desarrollo de este atributo, promedia un puntaje de 3,67 puntos de un total de 4,0 puntos.

Dicha competencia, involucra un nivel elevado de abstracción de estos conocimientos, pues la aplicación de estos, es de vital importancia a la hora de permitirle al Contador Público Auditor, desempeñarse eficientemente en el área financiera, permitiéndole lograr los objetivos de custodiar e invertir los valores y recursos de una empresa, mantener los sistemas de información adecuados para el control de activos y operaciones de dicha organización y proteger el capital invertido.

Puesto №7: En el séptimo lugar de la jerarquización evidenciada, es posible apreciar la competencia focalizada en la aplicación adecuada de las leyes, reglamentos y disposiciones tributarias en los trabajos asignados. El desarrollo de esta competencia, arroja una media de 3,59 puntos de un total de 4,0 puntos, ubicándose entre las dos medias más bajas entre las competencias evaluadas, pese a su valoración positiva.

El desarrollo esta competencia, es relevante en el ámbito tributario y en la disciplina en general, pues existen una serie de normativas y reglamentaciones que deben ceñirse a cabalidad. Es tal su grado de importancia, que el no aplicarlo de manera 
totalmente óptima, impide un buen desempeño en el asesoramiento fiscal y en la gestión administrativa.

Puesto N8: En el último lugar, se encuentra la competencia centrada en la aplicación adecuada de las normas de contabilidad y la participación en los procesos de generación de informe de contabilidad, la cual alcanza una media de 3,50 puntos de un total de 4,0 puntos.

Es importante mencionar, que si bien esta competencia es la que generó un promedio más bajo en comparación con las competencias anteriores, esto no quiere decir que no haya sido desarrollada satisfactoriamente, pero si habla de la necesidad de potenciarlas aún más.

A nivel global, es relevante señalar en este caso, que el desarrollo de las competencias profesionales, muestra una media de 3,71 puntos de un total de 4,0 puntos.

\section{DISCUSIÓN Y CONCLUSIONES.}

Al evaluar el desarrollo de competencias tanto transversales como profesionales en los 25 alumnos de Auditoría cohorte 2014 que cursaron su Práctica profesional, es posible aseverar, una evaluación positiva en 11 de las 13 competencias presentes en el perfil de egreso. Donde a nivel general, las competencias transversales, promediaron una media de 3,35 puntos de un total de 4,0 puntos, mientras las competencias profesionales, alcanzaron el rango más alto, promediando una media de 3,71 puntos. Esto lleva a inferir, que si bien la mayoría de las competencias fueron bien evaluadas, hay que prestar especial atención, a aquellos atributos de corte genérico.

Al adentrarse en el detalle por cada tipo de competencias, es factible dar cuenta, que mientras entre las competencias transversales más desarrolladas, se encuentran el compromiso ético por parte de los alumnos a la hora de actuar adecuadamente bajo principios morales en el ámbito profesional y personal, y la responsabilidad, al momento de cumplir con los dictámenes de la empresa, entre las menos desarrolladas, es posible encontrar, la adaptación al cambio y la autonomía e iniciativa personal. Estas dos últimas competencias evaluadas relativamente deficitarias, evidenciando la necesidad de una mayor intervención en ellas.

Es importante mencionar también, que la competencia de trabajo en equipo, la cual igualmente es evaluada en términos positivos, muestra una mejor valoración, en la variable orientada a la participación activa de los alumnos como ejecutor de las decisiones tomadas al interior del grupo, más que en la participación en discusiones que conducen a resoluciones. 
En el caso de las competencias profesionales por su parte, la evaluación da cuenta, que entre las competencias más desarrolladas se destaca en primer lugar la valoración que los alumnos le otorgan a la estructura orgánica de la empresa y a las normas y reglas por ella establecida, apuntando con esto, a una fortaleza en el área administrativa, y en segundo lugar, a la contribución que los alumnos generan en los procesos propios de la Auditoría, concretándose esto en la aplicación de los procedimientos necesarios para su ejecución.

Por otra parte, entre las competencias menos desarrolladas en comparación con las demás competencias profesionales, no obviando igualmente una valoración positiva de estas mismas, es posible encontrar en primer lugar, la aplicación adecuada de las leyes, reglamentos y disposiciones tributarias en los trabajos asignados, apuntando con esto, a la necesidad de potenciar de mejor manera la transferencia desde la teoría a la práctica en los conocimientos del área tributaria, y en segundo lugar, la aplicación adecuada de las normas de contabilidad y la participación en los procesos de generación de informes contables, evidenciándose así, el requerimiento de potenciar un rol más activo por parte del practicante entorno a esta área y a una mejor transferencia teórico-práctica, pero en el área de la contabilidad misma. No ha de llamar la atención entonces, que frente a esta última competencia, el hecho de distinguir los procesos económicos que se generan en las empresas y los criterios de registro contable, sea una competencia mejor evaluada que la aplicación misma de las normas de contabilidad y la participación en la elaboración de informes contables, pues el tema recae, en que mientras en la primera se exige un nivel taxonómico de comprensión, permitiendo visualizar los aspectos que deben ser considerados a la hora de evidenciar la realidad de una empresa, la otra requiere un nivel taxonómico mayor, pues está orientada a la aplicación de las normas de contabilidad a la hora de emitir un juicio y levantar informes contables.

Es relevante mencionar también, que entre aquellas competencias evaluadas de panera positiva pero que se posicionan en un rango medio en comparación con las demás, se destacan según orden de evaluación satisfactoria, en primer lugar, la valoración del rol e importancia que los alumnos le asignan a la información financiera en la empresa, seguido de la capacidad de distinguir los procesos económicos que se generan en las empresas y los criterios de registro contable, la incorporación de herramientas tecnológicas y por último, la demostración y aplicación de los conocimientos financieros.

En resumen, buscar medidas remediales que permitan seguir mejorando todos estos indicadores, es una tarea pendiente, donde el foco debe centrarse en el proceso de formación del Contador Público Auditor, donde la metodologías activas de enseñanza-aprendizaje, las evaluaciones alternativas, el asesoramiento y retroalimentación constante, la realización de prácticas tempranas y el potenciar las competencias por medio de distintas asignaturas, se vuelve relevante. 


\section{BIBLIOGRAFÍA.}

- CRUCH (2011). Innovación curricular en las universidades del consejo de rectores 2000- 2010. Proceso de Innovación Curricular.

- Gálvez E. (2013). Cuaderno de Apoyo Didáctico Metodología activa: favoreciendo los aprendizajes. Revista Construyendo juntos, Vol. №1, p.10.

- OIT (2012). ¿Qué es la competencia laboral? Recuperado http:// www.oitcinterfor.org/p\%C3\%A1 gina-libro/1-\%C2\%BFqu\%C3\%A9competencia-laboral

- Tobón S. (2007). El enfoque complejo de las competencias y el diseño curricular por ciclos propedéuticos. Revista de Acción Pedagógica, Vol. №16, p. 17.

- Tuning. (2007). Reflexiones y perspectivas de la Educación Superior en América Latina (Informe Final Proyecto Tuning América Latina 20042007). Bilbao, España: Publicaciones Universidad de Deusto, pp.37-71.

- Universidad de La Serena (2017). Plan de Estudios Carrera de Auditoría. Recuperado de http://www.userena.cl/boton-carreras/item/auditoria.html 
Anexos.

Anexo №1: Cuestionario de Evaluación Práctica Profesional.

\begin{tabular}{|c|c|c|c|c|c|c|}
\hline \multicolumn{7}{|l|}{ 1.- IDENTIFICACIÓN. } \\
\hline \multicolumn{7}{|l|}{ Nombre del estudiante: } \\
\hline \multicolumn{7}{|l|}{ Nombre de la empresa o Institución: } \\
\hline \multicolumn{7}{|l|}{ Nombre del Profesional a cargo del proceso: } \\
\hline \multicolumn{7}{|l|}{ Fecha de evaluación de la práctica: } \\
\hline \multirow[t]{2}{*}{ AREAS } & $\begin{array}{l}\text { Contabilida } \\
\text { dy costos }\end{array}$ & Tributación & Finanzas & Auditoria & $\begin{array}{l}\text { Sistemas de } \\
\text { información }\end{array}$ & Administarción \\
\hline & CONT & TRI & ค1 & AU & si & AD \\
\hline Indicador de desempeño. & । & $\begin{array}{c}\text { En desacuerdo } \\
\text { (1 ptos) }\end{array}$ & $\begin{array}{l}\text { Medianamente de } \\
\text { scuerso (2 pta) }\end{array}$ & $\begin{array}{l}\text { De acuerdo (3) } \\
\text { ptos) }\end{array}$ & $\begin{array}{l}\text { Totaimente de } \\
\text { scuerdo } 14 \text { ptos: }\end{array}$ & No cosservode. \\
\hline \multicolumn{7}{|l|}{$\begin{array}{l}\text { 1.- Demuestra responsabilidad en terminos de: cumplimiento de horarios. } \\
\text { asistencia y otras oblizaciones comprometidas. }\end{array}$} \\
\hline \multicolumn{7}{|l|}{$\begin{array}{l}\text { 2.- Participa en los diálogos y discusiones del equipo, desde sus ideas propias y/o } \\
\text { experiencias. }\end{array}$} \\
\hline \multicolumn{7}{|l|}{$\begin{array}{l}\text { 3.- Aporta información relevante, } y \text { atingente a los desafios que debe enfrentar et } \\
\text { equipo. }\end{array}$} \\
\hline \multicolumn{7}{|l|}{ 4.-Se involucra cuando surge una situación relevante. } \\
\hline \multicolumn{7}{|l|}{ 5.- Muestra ideas claras y determinante frente al cambio. } \\
\hline \multicolumn{7}{|l|}{$\begin{array}{l}\text { 6.- Muestra un compromiso ettico para con su disciplina y para con el entorno en } \\
\text { el cual trabaja. }\end{array}$} \\
\hline \multicolumn{7}{|l|}{ 1.- Demuestra conocimientos en las áreas en las cuales se desempeña. } \\
\hline \multicolumn{7}{|l|}{$\begin{array}{l}\text { 2.- Distinque los procesos económicos que se generan en las empresas } \\
\text { (instituciones) y los criterios de repistro contable. }\end{array}$} \\
\hline \multicolumn{7}{|l|}{$\begin{array}{l}\text { 3.- Aplica las normas de contabilidad y participa en los procesos de generación de } \\
\text { informes de contabilidad. }\end{array}$} \\
\hline \multicolumn{7}{|l|}{$\begin{array}{l}\text { 4- Valora el rol e importancia de la información financiera en la empresa y su } \\
\text { utllidad en la toma de decisiones. }\end{array}$} \\
\hline \multicolumn{7}{|l|}{$\begin{array}{l}\text { 5. Aplica adecuadamente las leves, reglamentos y disposiciones tributarias en los } \\
\text { trabajos asignados. }\end{array}$} \\
\hline \multicolumn{7}{|l|}{$\begin{array}{l}\text { 6.- Contribuve en los procesos de Auditoria y aplica los procedimientos necesarios } \\
\text { para su ejecusión. }\end{array}$} \\
\hline \multicolumn{7}{|l|}{$\begin{array}{l}\text { 7. Demuestra y aplica conocimientos del área financiera en el desempeño de su } \\
\text { práctica. }\end{array}$} \\
\hline \multicolumn{7}{|l|}{$\begin{array}{l}\text { 8.- Valora la importancia de la estructura orzánica de empresa (institución), y } \\
\text { demuestra compromiso con el cumplimiento de las normas y procedimientos de la } \\
\text { organización. }\end{array}$} \\
\hline $\begin{array}{l}\text { 9- Incorpora herramientas tecnológicas y las utiliza de manera eficiente para ei } \\
\text { desempeȟo de su trabajo. }\end{array}$ & & & & & & \\
\hline
\end{tabular}

Fuente: Escuela de Auditoría. 
Revista de Investigación Aplicada en Ciencias Empresariales 